\title{
A complexidade do custo socioeconômico da filariose linfática
}

\author{
The complexity of the socioeconomic costs of lymphatic filariasis
}

\author{
Denise Mattos ${ }^{1,2}$ e Gerusa Dreyer ${ }^{1,2,3}$
}

\begin{abstract}
RESUMO
A falta de conhecimento do impacto socioeconômico das diversas doenças é, geralmente, um dos maiores obstáculos para a obtenção de verbas para investimentos em pesquisa aplicada, assim como para iniciar a implementação de programas de controle necessários aos países em desenvolvimento. Os autores analisam e ressaltam aspectos relevantes da complexidade de quantificação do impacto socioeconômico da filariose linfática e enfatizam as lacunas existentes em algumas áreas do conhecimento, que ainda não foram devidamente exploradas para os portadores da doença. Aos dados de literatura aliam os ecos do aprendizado adquirido através do atendimento de pacientes portadores de infecção e doença bancroftiana em serviço terciário de referência do Núcleo de Ensino Pesquisa e Assistência em Filariose (NEPAF), Recife, Brasil. 0 impacto social, no seu sentido mais abrangente, mostra indícios fortes de perdas na qualidade de vida dos pacientes, infelizmente ainda pouco documentadas e, até certo ponto, de muito difícil contabilização, pelo tão peculiar abandono social das comunidades endêmicas.
\end{abstract}

Palavras-chaves: Filariose bancroftiana. Custo social. Custo econômico. Controle. Linfedema. Hidrocele.

\begin{abstract}
Lack of knowledge of the socioeconomic impact of various diseases is generally one of the biggest obstacles to obtaining funds for investment in applied research and for starting to implement control programs that are needed in developing countries. The authors analyze and emphasize important aspects of the complexity of quantifying the socioeconomic impact of lymphatic filariasis. They highlight the gaps that exist within some fields of knowledge and that these gaps have still not been properly explored with regard to individuals with this disease. They bring together the data in the literature and echoes from their own experience gained through caring for patients with Bancroftian infection and disease at a tertiary referral service (NEPAF - Filariasis Care, Research and Teaching Center, Recife, Brazil). The social impact, in its broadest sense, consists of strong indications of losses of quality of life among patients. Unfortunately, this is still poorly documented and, up to a certain point, very difficult to quantify because of the very particular social abandonment of the endemic communities.
\end{abstract}

Key-words: Bancroftian filariasis. Social cost. Economic cost. Control. Lymphedema. Hydrocele.

Entre as oito filarioses que afetam o ser humano, a linfática (causada pela Wuchereria bancrofti e pelas Brugias) é, sem dúvida, a mais importante, pela grandeza de seus números. Estima-se que, aproximadamente, 120 milhões de indivíduos sejam portadores de microfilárias circulantes, porém ainda sem apresentarem doença clínica, distribuídos em 83 países de clima tropical e subtropical ${ }^{44}$. A prevalência da infecção aumenta durante a infância e tende a se estabilizar no início da fase adulta e é mais alta entre os indivíduos do sexo masculino e na população de 20 a 40 anos de idade. Embora a distribuição da infecção pareça global, aproximadamente um terço dos indivíduos infectados reside na Índia, outro terço na África e o restante se encontra, predominantemente, na região ocidental do Pacífico e no sudeste da Ásia. As Américas representam 0,3\% da prevalência global e o país de maior número de casos é o Haiti, seguido pela
República Dominicana, pela Guiana Inglesa e pelo Brasil. Neste, há focos considerados de transmissão ativa no Grande Recife, em Pernambuco $^{23}$, e na Cidade de Maceió, em Alagoas ${ }^{11}$. Belém do Pará, que na década de 1950 era a área de maior prevalência, hoje é considerada um foco em extinção ${ }^{10}$. A doença clínica é um duro encargo social e econômico para os seus portadores e para o Estado. Estima-se um número global de 40 milhões de portadores das duas formas mais prevalentes da doença, a hidrocele e o linfedema ${ }^{26}$. Há cerca de 16 milhões de portadores de linfedema ${ }^{15}$, e a Índia, sozinha, seria responsável por mais de 7 milhões de indivíduos ${ }^{31}$. Entre os portadores de linfedema de todos os países endêmicos não há qualquer dado sobre quanto esse número representaria de portadores de elefantíase. A endemia se instala nas comunidades de forma lenta e silenciosa e, muitas vezes, mantém os casos mais deformantes escondidos em suas

1. Serviço Social, Hospital das Clínicas, Universidade Federal de Pernambuco, Recife, PE. 2. Organização Não-governamental Amaury Coutinho para Doenças Endêmicas e Tropicais, Recife, PE. 3. Centro de Pesquisas Aggeu Magalhães, Fundação Oswaldo Cruz, Recife, PE.

Órgão financiador: Organização Não-governamental Amaury Coutinho, com os custos do processo na preparação do manuscrito e no envio para a revista.

Endereço para correspondência: Dra. Denise Mattos. Organização Não Governamental Amaury Coutinho para Doenças Endêmicas e Tropicais. R. Conselheiro Portela 665/sala 120, Graças, 52020-030 Recife, PE.

Telefax: $55813426-4348$.

e-mail: mattos.denise@gmail.com

Recebido para publicação em 18/06/2008

Aceito em 30/06/2008 
casas e afastados do sistema de detecção da saúde pública local. Como não é uma doença letal, em muitos dos países endêmicos, a filariose não lidera a atenção da mídia nem das políticas públicas, como o fazem doenças com alta mortalidade, como a malária, a síndrome da imunodeficiência adquirida (SIDA) e a desnutrição. Por outro lado, ela traduz, para os portadores das formas crônicas, um custo individual importante ainda não devidamente contabilizado e que interfere negativamente na qualidade de vida dos pacientes e de seus familiares ${ }^{5621}$.

A maioria dos países endêmicos não possui evidências bem documentadas das perdas de produção nas economias local e individual ou familiar, nem dos custos governamentais. No entanto, nos últimos anos, observa-se uma maior preocupação em se quantificar o impacto sociocultural da doença ${ }^{46}, 0$ que fortalece a caracterização da filariose como um problema de saúde pública, de acordo com a Assembléia Mundial da Saúde, em $1997^{42}$. Ainda assim, existe uma importante subestimação do problema, devido, inicialmente, à falta de dados atualizados das prevalências reais das principais formas crônicas na grande maioria das áreas endêmicas. 0 último trabalho que aborda o assunto, e de forma global, foi publicado há uma década ${ }^{25}$.

\section{OS CUSTOS DIRETOS}

As informações sobre as cifras gastas pelos cofres públicos nos diversos países são limitadas. Em 1975, na Tanzânia, um hospital distrital constatou que $15 \%$ das cirurgias consideradas de maior porte foram identificadas como hidrocelectomias ${ }^{39}$. No Brasil, não existe nenhum dado oficial sobre o custo governamental no atendimento dos casos cirúrgicos ou clínicos, quer em assistência primária ou terciária (G Dreyer: comunicação pessoal). Por outro lado, em muitas regiões endêmicas, principalmente da Ásia e da África, corre-se o risco de que esses custos sejam erroneamente estimados como muito baixos ou mesmo inexistentes. Várias razões poderiam ser aventadas. Entre elas, a falta de disponibilidade dos serviços públicos, fato comum em muitas localidades dos países endêmicos, a falta de condições dos habitantes para custearem os procedimentos ou, até mesmo, a dificuldade de transporte até a unidade de saúde mais próxima.

A Organização Mundial de Saúde (OMS) contabiliza ser a filariose linfática a segunda causa de incapacidade permanente no mundo ${ }^{41}$, perdendo somente para os distúrbios da afetividade, grupo em que, provavelmente, estão também os milhares de pacientes com as formas mais estigmatizantes da doença ${ }^{421}$. Existem evidências bem consolidadas de que o estágio crônico reduz, sobremaneira, a condição produtiva/laboral de seus portadores ${ }^{9} 1639$. Há quem argumente, entretanto, que esse impacto econômico é, de alguma maneira, reduzido pela justificativa de a maioria das pessoas incapacitadas apresentarem a doença crônica após a faixa etária produtiva laboral ${ }^{40}$. Isso, porém, não parece verdadeiro para muitas comunidades com alto índice de transmissão, quando a hidrocele acomete uma parcela muito jovem de sua populaçã $0^{14}$. No Grande Recife, área endêmica de filariose bancroftiana, a idade média de portadores de hidrocele filarial avaliados em dois estudos foi de $35,8 \operatorname{anos}^{19}$ e de 24,2 anos $^{29}$.
$\mathrm{Na}$ Índia e no continente africano, estima-se que a perda anual da produtividade devido à filariose linfática chegue a um bilhão de dólares americanos. Estima-se também que, anualmente, mais de 10 milhões de pessoas busquem tratamento, gerando despesas que excedem 30 milhões de dólares americanos para os mais diversos itens - transporte, medicamentos, pagamento da consulta, custos do acompanhante e de acomodação. As despesas do acompanhante e de acomodação são de grande importância devido ao número de portadores residentes em área rural. Foi calculado que, do montante de 30 milhões acima referidos, cerca de 23 milhões foram gastos pelos pacientes do sexo masculino e 8 milhões pelos do sexo feminino, não sendo incluído qualquer custo governamental ${ }^{31}$.

Como curiosidade, o impacto individual e econômico da filariose não está restrito somente aos governos e aos habitantes das áreas endêmicas. Durante a II Guerra Mundial, um grande número de soldados americanos se infectou nas ilhas do Pacífico e a capacidade de luta dos militares ficou extremamente comprometida, com uma incidência de adenite e de linfangite (ambas causadas pela morte dos vermes adultos através de uma hiper-resposta imunológica, característica do hospedeiro não endêmico) que variou de 55 a $65 \%$ em alguns batalhões. 0 comando geral norte-americano ordenou, então, a evacuação de todas as suas unidades militares das ilhas do Pacífico. Napier estimou que o custo dessa operação filarial para as forças armadas americanas foi de, aproximadamente, 100 milhões de dólares ${ }^{28}$.

\section{OS CUSTOS INDIRETOS}

Na realidade, há uma magnitude médico-social e política complexa de ser dimensionada, muitas vezes pelo desconhecimento de particularidades do que acontece com o portador de formas crônicas da filariose $e^{4}$. Suas perspectivas parecem sombrias quanto ao uso dos mais elementares direitos de cidadania que são, entre outros, a oportunidade ao trabalho e ao seguro social, à moradia e à participação social. A incorporação dos aspectos sociais nos prontuários dos pacientes atendidos pelo serviço terciário de referência para filariose em Recife (NEPAF - Núcleo de Ensino Pesquisa e Assistência em Filariose), seguindo-se à consulta médica, veio ratificar essa falta de perspectivas de forma muito clara. Em uma amostra inicial de 50 homens e 50 mulheres portadores de formas crônicas, foi constatado que 19 (38\%) homens e 17 (34\%) mulheres não tinham qualquer atividade laboral e não conseguiram inclusão no amparo social da LOAS (Lei Orgânica da Assistência Social) ${ }^{18}$, apesar de apresentarem uma renda per capita menor que $1 / 4$ do salário mínimo exigido pela lei (D Mattos: comunicação pessoal). Trabalho recente em Recife relatou diversos aspectos de pacientes do sexo feminino portadoras de elefantíase; ficou demonstrado o sentimento de perda na qualidade de vida e do abandono pelos familiares e pelo estado ${ }^{2}$. Em Togo, achados similares de sentimento de abandono foram relatados em um estudo com 188 portadores de linfedema: $17 \%$ relataram ser evitados pelos familiares e $36 \%$ pela comunidade ${ }^{34}$. Um trabalho do sudeste da Tanzânia, distrito 
de Rufiji, relatou que os portadores de elefantíase (com e sem episódios agudos bacterianos) e de hidrocele limitaram, em muito, a sua participação nas atividades sociais da comunidade ${ }^{27}$. Em qualquer sociedade, seja ou não capitalista, a perda da capacidade laborativa por causa da doença altera toda a dinâmica familiar, muitas vezes, provocando a inversão de papéis que a cultura já demarcou. Os autores acreditam que os sentimentos de exclusão social e de vergonha devem se somar à tendência de isolamento desses cidadãos e, mais uma vez, aumentando os custos que dificilmente podem ser quantificáveis. Isso ressalta, duplamente, a complexidade do contexto socioeconômico dos portadores da doença. Independentemente da doença crônica deformante, seus portadores continuarão com o papel e a missão de responder às suas necessidades básicas e às de seus dependentes. Que custo maior poderia existir para uma determinada comunidade ou um país endêmico do que ver inutilizados os seus cidadãos? Mais do que pela falta de escolarização ou experiência de trabalho, as portas do mercado se fecham rapidamente quando existe a visibilidade das formas crônicas. Quando conseguem trabalhar, são explorados, como demonstrado por Ramu e cols $^{32}$, ao compararem o salário (ou o ganho) entre portadores e não portadores de filariose pareados por faixa etária: há uma redução de quase $30 \%$ entre os acometidos pela doença ${ }^{32}$. Na maioria das famílias com membros afetados pela doença, há, freqüientemente, a necessidade de se contratar mão-de-obra externa, em detrimento a outras despesas, ou ter apoio para os serviços domésticos. Além de aumentar a despesa com o item doença, o fato promove um impacto negativo importante na renda per capita familiar e/ou na auto-estima da dona-de-casa ao se perceber incapaz de exercer o papel social para o qual foi, provavelmente, criada ${ }^{21}$. Kessel ${ }^{16}$, em 1957, já descrevia que, na Polinésia Francesa, a vida produtiva dos portadores de hidrocele e de elefantíase era bastante reduzida e os mesmos se tornavam um fardo para os familiares. Em Gana, a média anual de dias perdidos de trabalho pelos episódios agudos bacterianos em portadores de linfedema foi de três dias por pessoa. Inicialmente, esse período pode não parecer muito longo, mas, como a maioria dos episódios ocorre durante o inverno, época de maior produção agrícola, a perda individual e familiar é importante porque muitas pessoas não produzem qualquer alimento durante aquele ano ${ }^{14}$. Em Recife, um estudo feito com 119 pacientes portadores de linfedema mostrou que, em média, anualmente ocorrem 3,6 episódios agudos, o que implica a incapacidade transitória de, pelo menos, 45 dias por ano ${ }^{1}$.

\section{A ELIMINAÇÃo dA FILARIOSE}

O Programa de Eliminação da Filariose Linfática, lançado no ano 1999 pela Organização Mundial da Saúde ${ }^{43}$, está estruturado em dois importantes pilares: a interrupção da transmissão pelo tratamento em massa das comunidades endêmicas e o controle da morbidade (aliviar o sofrimento e prevenir a incapacidade) ${ }^{36}$. 0 sucesso da aderência dos países comprometidos com 0 mapeamento da infecção pode ser traduzido pelo lançamento do Programa Global de Eliminação da Filariose Linfática em 42 dos
83 países endêmicos, cobrindo uma população de mais de 610 milhões em $2005^{45}$. Por outro lado, o mapeamento/quantificação da doença crônica está, ainda, em uma fase incipiente. É imperativo que, globalmente, a implementação do controle de morbidade caminhe em paralelo ao controle da transmissão para atender à expectativa gerada nas populações endêmicas, ou seja, prevenir e aliviar o seu sofrimento ${ }^{3}$, pois é essa a visibilidade do agravo para elas, uma vez que a microfilaremia raramente produz doença clínica ${ }^{24}$. Nesse sentido, Kumari e cols ${ }^{17}$ alertam para a necessidade de se investigar, de forma multidimensional, a percepção do paciente sobre os impactos: 1) da doença em sua vida; 2) do tratamento recebido; 3) das mudanças nas suas funções psicológicas e sociais e 4) do seu bem-estar, como indicadores cruciais para avaliar os custos antes e depois da implementação dos programas de morbidade ${ }^{17}$.

Recentemente, trabalho publicado por Goldamn e cols ${ }^{13} \mathrm{com}$ dados obtidos de sete países endêmicos mostrou que a eliminação da filariose, enquanto infecção, é um processo relativamente de baixo custo, quando comparado a outros programas de saúde pública, e que variou de US\$0.06 a 2.23 por pessoa por ano. Essa variação dependeu do uso de voluntários (fator de maior relevância), do tamanho da população tratada e da experiência dos países com o uso de tratamento em massa antifilarial ${ }^{13}$. De interesse, o custo/benefício da eliminação da filariose na Índia já foi calculado, em 1971, como sendo cerca de 133 milhões de dólares americanos por ano ${ }^{30}$, quase 30 anos antes da divulgação oficial, em 1997, do programa global da eliminação da filariose ${ }^{42}$. Apesar de inicialmente parecer uma grande cifra, os benefícios econômicos de programas de controle parecem compensar, quando se exemplifica o que foi obtido na China (Cidade de Miaoxi): para cada dólar americano gasto houve um benefício líquido de 5,75 dólares ${ }^{37}$.

Trabalho pioneiro em Jaboatão - Pernambuco ${ }^{22}$, constatou as péssimas condições socioeconômicas de crianças/adolescentes portadores de microfilaremia e de seus familiares. Esse estudo permitiu ratificar que, independentemente da implementação dos dois pilares (transmissão/morbidade), o impacto social somente acontecerá se existirem ações concretas, seqüenciadas e articuladas entre as secretarias municipais de saúde e de meio ambiente, educação, política de geração de emprego e de assistência social. Com muita pertinência, Vasconcelos ${ }^{38}$ descreve: o campo da saúde é o campo da vida e, por isso mesmo, está relacionado diretamente com a economia, com o trabalho, com a cultura, com a habitação, com o lazer, com a educação, com a política e com a ética. Como fazer esse conceito ter ressonância nas comunidades que abrigam os portadores da forma crônica da doença filarial e de suas complicações e ser contabilizado nos custos do agravo filarial? O desenvolvimento da economia local, através do papel social das empresas, seria uma das formas de se reduzir a transmissão da filariose. A Guiana Inglesa é um exemplo disso. Foi possível documentar a redução da prevalência da infecção filarial a partir da visão do papel social das empresas que implementaram ações capazes de melhorar as condições de habitação, do suprimento de água e do esgotamento sanitário nas plantações de cana ${ }^{12}$. Por outro 
lado, alguns projetos sociais podem favorecer a transmissão da filariose, aumentando a médio e longo prazos os custos socioeconômicos da doença, uma vez que as formas clínicas, em muitos casos, aparecem vários anos depois de instalada a infecção ${ }^{6}$. Exemplo desse processo ocorreu no Sri Lanka, com a implantação de produtos da fibra do coco, que aumentou os criadouros de vetores ${ }^{2035}$.

\section{O PROBLEMA, A ALTERNATIVA}

Um dos maiores obstáculos para a obtenção de verbas para programas de controle e de pesquisas aplicadas na área de saúde dos países em desenvolvimento é a falta da noção real do impacto social e econômico das diversas doenças. Geralmente, o questionamento que inicia o processo envolve o seguinte aspecto: quão importante é uma dada doença sob o ponto de vista da saúde pública para uma determinada região ou um país? Estudos bem desenhados, que forneçam dados robustos (positivos ou negativos), proporcionam, obviamente, uma maior clareza no escalonamento das prioridades, a partir da extensa lista de problemas que os países em desenvolvimento têm que priorizar. A clareza e a confiabilidade dos dados são essencialmente determinantes não apenas para o planejamento, mas também para a realização de um trabalho profissional/ governamental de qualidade. Demonstrar e fazer constar em cifras a carga socioeconômica de uma determinada região endêmica para filariose linfática ajudará a dar a visibilidade necessária para colocá-la na lista de prioridades governamentais. 0 impacto econômico da filariose linfática, em linhas gerais, antes e depois da intervenção, pode ser agrupado em vários níveis que facilitariam a coleta de dados: 1) o custo direto na economia familiar (por exemplo, contrato de ajudantes, transporte, acomodação - nas áreas de filariose rural - compra de medicamentos e gastos com cirurgias e hospitalizações, principalmente em países que não têm um serviço público de saúde; 2) a perda individual da produtividade e a repercussão para a economia de cada microrregião; 3) o custo governamental na manutenção da infra-estrutura de clínicas e de hospitais terciários para atender os portadores da doença; 4 ) as perdas individuais pelas amplas repercussões na qualidade de vida. Os autores sugerem que a sistematização dos dados socioeconômicos da população atendida poderia dar maior qualidade à apreensão do objeto de trabalho, melhor conhecimento do perfil socioepidemiológico e das maiores necessidades sociais de uma dada comunidade endêmica. Contribuiria também para reforçar as cifras das perdas econômicas e aumentar a visibilidade de uma doença que se instala de forma insidiosa e sempre silenciosa, por não ser capaz de acontecer em forma epidêmica. Ao mesmo tempo, a sistematização no acesso do conhecimento para a população e da populaçã̃ $0^{33}$ permitiria envolver a educação para a prevenção e sinalizar para a possibilidade do controle social das ações políticas em relação à infra-estrutura determinante da transmissão: habitação e saneamento básico ${ }^{22}$. Isso seria particularmente interessante no Brasil, pois rapidamente se poderia avaliar a grandeza do problema e implementar o programa de controle de morbidade ${ }^{36}$, em paralelo ao controle da transmissão, baseado na real demanda encontrada no Grande Recife (PE) e em Maceió (AL), as duas únicas áreas endêmicas brasileiras. Visto dessa maneira, o Brasil encontra-se em situação privilegiada para eliminar um agravo importante da extensa lista de seus problemas de saúde pública.

\section{CONSIDERAÇÕES FINAIS}

Como enfatizado por Durrheim e cols ${ }^{8}$, a associação entre filariose linfática e pobreza é evidente, quando $94 \%$ dos países com os mais baixos índices de desenvolvimento humano (IDH) são endêmicos ${ }^{8}$. Isso antecipa a compreensão da dificuldade (ou mesmo da impossibilidade) de esses países implementarem o programa de morbidade sem ajuda não-governamental. Cabe aos pesquisadores dos países endêmicos gerar as informações adequadas sobre os custos socioeconômicos da filariose linfática, pois esse conhecimento teria um papel fundamental em atrair investidores para lutar por uma boa causa social, mostrando em cifras o custo-beneficio do processo.

\section{AGRADECIMENTOS}

Os autores agradecem o apoio financeiro e logístico da Organização Não Governamental Amaury Coutinho para Doenças Endêmicas e Tropicais no preparo do manuscrito para publicação.

\section{REFERÊNCIAS}

1. Dreyer G, Addiss D, Gadelha P, Lapa E, Williamson J, Dreyer A. Interdigital skin lesions of the lower limbs among patients with lymphoedema in an area endemic for bancroftian filariasis. Tropical Medicine and International Health 11: 14751481, 2006.

2. Dreyer G, Mattos D. Perspectives of elephantiasis in an endemic area of Brazil. Journal of Lymphoedema 2: 24-31, 2007

3. Dreyer G, Mattos D. A Prevenção da elefantíase em áreas endêmicas de bancroftose: realidade ou utopia? Prática Hospitalar 9: 94-96, 2007.

4. Dreyer G, Mattos D, Norões J. Filariose bancroftiana. In: Coura JR (ed) Dinâmica das doenças infecciosas e parasitárias. Guanabara Koogan, Rio de Janeiro, p. 1087-1106, 2005.

5. Dreyer G, Norões J. Filariose bancroftiana: o reverso das alterações orgânicas. Jornal Brasileiro de Psiquiatria 47: 227-231, 1998.

6. Dreyer G, Norões J, Addiss D. The silent burden of sexual disability associated with lymphatic filariasis. Acta Tropica 63: 57-60, 1997.

7. Dreyer G, Norões J, Figueredo-Silva J. Elimination of lymphatic filariasis as a public health problem. New insights into the natural history and pathology of bancroftian filariasis: implications for clinical management and filariasis control programs. Transactions of the Royal Society of Tropical Medicine and Hygiene 94: 594-596, 2000

8. Durrheim DN, Wynd S, Liese B, Gyapong JO. Lymphatic filariasis endemicity - an indicator of poverty? Tropical Medicine and International Health 9: 843-845, 2004 .

9. Evans DB, Gelband H, Vlassoff C. Social and economic factors and the control of lymphatic filariasis: a review. Acta Tropica 53: 1-26, 1993.

10. Fontes G, Braun RF, Fraiha Neto H, Vieira JBF, Padilha SS, Rocha RC, Rocha, EMM. Filariose linfática em Belém, Estado do Pará, Norte do Brasil e a perspectiva de 
eliminação. Revista da Sociedade Brasileira de Medicina Tropical 38: 131-136, 2005.

11. Fontes G, Rocha EMM, Brito AC, Antunes CMF. Lymphatic filariasis in Brazilian urban area (Maceió, Alagoas). Memórias do Instituto Oswaldo Cruz 93: 705-710, 1998.

12. Giglioli G, Beadnell HM. Filariasis in British Guiana. Some industrial medical problems. Indian Journal of Malariology 14: 651-658, 1960.

13. Goldman AS, Guisinger VH, Aikins M, Amarillo ML, Belizario VY, Garshong B, Gyapong J, Kabali C, Kamal HA, Kanjilal S, Kyelem D, Lizardo J, Malecela M, Mubyazi G, Nitièma PA, Ramzy RM, Streit TG, Wallace A, Brady MA, Rheingans $\mathrm{R}$, Ottesen EA, Haddix AC. National mass drug administration costs for lymphatic filariasis elimination. Plos Neglected Tropical Diseases 1: e67, 2007.

14. Gyapong JO, Gyapong M, Evans DB, Aitkins MK, Adjei S. The economic burden of lymphatic filariasis in Northern Ghana. Annals of Tropical Medicine and Parasitology; 90: 39-48, 1996.

15. Jamal S, Pani SP. Long term follow up of filarial edema with conservative medical management. Lymphology 33(supl): 144-146, 2000.

16. Kessel JF. Disabling effects and control of filariasis. The American Journal of Tropical Medicine and Hygiene 6: 402-414, 1957.

17. Kumari AK, Krishnamoorthy K, Harichandrakumar K, Das L. Health related quality of life, an appropriate indicator to assess the impact of morbidity management and disability prevention activities towards elimination of lymphatic filariasis. Filaria Journal 6: 8, 2007.

18. Lei Orgânica da Assistência Social (LOAS). Lei $n^{0} 8742$, de 07/12/1993. Diário Oficial da União, Brasília, DF, publicado em 08/12/1993.

19. Maciel AFF. Ocorrência de infecção filarial em portadores de hidrocele moradores de área endêmica para bancroftose. Dissertação de Mestrado, Universidade Federal de Pernambuco, Recife, PE, 2005.

20. Mak JW. Problems in filariasis control and the need for human behavior and socio-economic research. The Southeast Asian Journal of Tropical Medicine and Public Health 17: 479-485, 1986.

21. Mattos D, Dreyer G. Elefantíase em área de filariose bancroftiana: o lado humano da doença. Revista de Patologia Tropical 35: 117-124, 2006.

22. Mattos D, Mota S, Dreyer G. Aspectos da realidade social de crianças e adolescentes atendidos em serviço de referência para filariose bancroftiana - Recife, Estado de Pernambuco. Revista da Sociedade Brasileira de Medicina Tropical 41: 29-35, 2008.

23. Medeiros Z, Dreyer G, Andrade LD, Pires ML, Mendes J, Pimentel R. Wuchereria bancrofti microfilarial density of autochthonous cases and natural Culex infectivity rates in Northeast Brazil. The Journal of Tropical Medicine and Hygiene 95: 214-217, 1992

24. Medeiros Z, Gomes J, Beliz F, Coutinho A, Dreyer P, Dreyer G. Screening of army soldiers for Wuchereria bancrofti infection in metropolitan Recife region, Brazil: implications for epidemiologic surveillance. Tropical Medicine and International Health 4: 499-505, 1999.

25. Michael E, Bundy DA. Global mapping of lymphatic filariasis. Parasitology Today 13: 472-476, 1997

26. Michael E, Bundy DA, Grenfell BT. Re-assessing the global prevalence and distribution of lymphatic filariasis. Parasitology 112: 409-428, 1996.

27. Mujinja PGM, Gasarasi DB, Premji ZG, Nguma J. Social and economic impact of lymphatic filariasis in Rufiji district, Southeast Tanzania. Lymphatic filariasis research and control in Africa: report on a workshop 69 held in Tanga, Tanzania. Danish Bilharziasis Laboratory, Denmark and National Institute for Medical Research, Tanzania, 1997.

28. Napier LE. Filariasis in U.S. marines. The Journal of Tropical Medicine and Hygiene 50: $189,1947$.
29. Norões J. Aspectos clínicos, parasitológicos e histopatológicos decorrentes da morte de vermes adultos de Wuchereria bancrofti em linfáticos intra-escrotais Tese de Doutorado. Universidade Federal de São Paulo, São Paulo, SP, 2000.

30. Prescott NM. On the benefits of tropical disease control. In: Wood C, Rue Y (eds) Health policies in developing countries. Royal Society of Medicine. International Congress and Symposium Series. p. 35-37, London, 1980.

31. Ramaiah KD, Das PK, Michael E, Guyatt H. The economic burden of lymphatic filariasis in India. Parasitology Today 16: 251-253, 2000.

32. Ramu K, Ramaiah KD, Guyatt H, Evans D. Impact of lymphatic filariasis on the productivity of male weavers in a south Indian village. Transactions of the Royal Society of Tropical Medicine and Hygiene 90: 669-670, 1996.

33. Rath K, Nath N, Shaloumy M, Swain BK, Suchismita M, Babu BV. Knowledge and perceptions about lymphatic filariasis: a study during the programme to eliminate lymphatic filariasis in an urban community of Orissa, India. Tropical Biomedicine 23: 156-162, 2006.

34. Richard SA, Mathieu E, Addiss DG, Sodahlon YK. A survey of treatment practices and burden of lymphoedema in Togo. Transactions of the Royal Society of Tropical Medicine and Hygiene 101: 391-397, 2007.

35. Schweinfurth U. Filarial diseases in Ceylon: a geographic and historical analysis Ecology of Disease 2: 309-319, 1983.

36. Seim AR, Dreyer G, Addiss D. Controlling morbidity and interrupting transmission: twin pillars of lymphatic filariasis elimination. Revista da Sociedade Brasileira de Medicina Tropical 32: 325-328, 1999

37. Shin Z, Sun D, Shi H. Cost-benefit analysis on malayan filariasis control in Miaox township, Huzhou city, Zhejiang province during 1964-1987. Chinese Journal of Parasitology and Parasitic Diseases 13: 193-196, 1995.

38. Vasconcelos AM. Para além da escolha de Sofia: tendências atuais da prática profissional dos assistentes sociais na rede de saúde do município do Rio de Janeiro. In: Vasconcelos AM (ed) A prática do serviço social: cotidiano, formação e alternativas na área da saúde. $2^{\text {a }}$ edição, Editora Cortez, São Paulo, p. 413-507, 2003 .

39. Wegesa P, McMahon JE, Abaru DE, Hamilton PJ, Marshall TF, Vaughan JP. Tanzania filariasis project: survey methodology and clinical manifestations of bancroftian filariasis. Acta Tropica 36: 369-377, 1979.

40. Wijers DJB. Bancroftian filariasis in Kenya I. Prevalence survey among adult males in the Coast Province. Annals of Tropical Medicine and Parasitology 71: 313-331, 1977.

41. World Health Organization. The world health report 1995: bridging the gaps. Geneva, 1995. Disponível em: <http://www.who.int/whr/1995/en/index.html> (Acessado em: 7 jun, 2008)

42. World Health Organization. Elimination of lymphatic filariasis as a public health problem. Fiftieth World Health Assembly. WHA 50.29. Resolution of the executive board of the WHO. Geneva, 1997. Disponível em: <www.who.int/entity/lymphatic_ filariasis/resources/WHA_50\%2029.pdf> (Acessado em: 7 jun, 2008).

43. World Health Organization. Building partnership for lymphatic filariasis: strategic plan. WHO/FIL/99.198. Geneva, 1999. Disponível em: <http://whqlibdoc.who. int/hq/1999/WHO_FIL_99.198.pdf> (Acessado em: 7 jun, 2008).

44. World Health Organization. Global programme to eliminate lymphatic filariasis annual report on lymphatic filariasis 2003. WHO/CDS/CPE/CEE/2005.52 Geneva, 2005. Disponível em: <whqlibdoc.who.int/hq/2005/WHO_CDS_CPE_CEE 2005.52.pdf> (Acessado em: 7 jun, 2008).

45. World Health Organization. Global programme to eliminate lymphatic filariasis. Weekly Epidemiological Record 81: 221-232, 2006. Disponível em: < http://www. who.int/wer/2006/wer8122/en/index.html> (Acessado em: 7 jun, 2008).

46. Wynd S, Melrose WD, Durrheim DN, Carron J, Gyapong M. Understanding the community impact of lymphatic filariasis: a review of the sociocultural literature. Bulletin of the World Health Organization 85: 493-498, 2007. 